\title{
Research on Hand-Held Insulation Tool Detection Alarm Device of Power Operation
}

\author{
Nan Li \\ North China Electric Power University, Baoding, Hebei, China \\ 710575066@qq.com
}

Keywords: Insulation monitoring; Automatic alarm; Safety specification; Positioning communication; Electric power operation

\begin{abstract}
In the power operation, according to safety regulations, the staff must wear insulated gloves. However, in practice, the staff is easy to overlook, therefore, to improve security, we have developed a hand-held insulation tool detection alarm device. Method: Device using k60 microcontroller, microcontroller with ADC module that can detect the battery voltage, the other in the K60 microcontroller external TSI module, TSI module with a copper wire connected to the wire, you can distinguish between naked hand contact with insulated gloves, if the staff did not The TSI module can detect and send the signal to the GPS module. The module locates the workplace, and the voice module issues a warning. The LED flashes and the device uploads the data through the GPRS module. When the alarm device, when wearing insulated gloves for grounding operation, the gate circuit does not turn on, the light-emitting diode does not shine, workers can operate normally; when the empty-handed operation, the gate circuit, diode light, honey began ringing, Prompt the operator to wear insulated gloves.
\end{abstract}

\section{Introduction}

Now the rapid development of power industry, power security is more and more important. In the electric power operation, the worker carries on the line maintenance time, in order to guarantee the personal safety, all needs to cut off electricity first, uses the electroscope to examine the electricity, then uses the earth line to earth, then carries on the corresponding maintenance. Workers often use a variety of safety insulation tools, such as insulation rods, grounding rods, electroscope, etc., these tools not only play a role in the completion of the work, but also to protect personal safety plays an important role. According to safety standards, workers in the use of insulated tools, should be hand-insulated gloves, but in practice, workers easily overlooked safety norms, direct hand to pick up, it is likely to cause electric shock and other phenomena.

At present there is no similar market applied to the power of hand-held insulation tools, alarm detection devices. Such as the widely used high-voltage ground wire, operating personnel installed, if not pay attention to the operating procedures, direct hand-installed ground wire, there may be an electric shock induction. The common ground wire insulation rod, insulated by the operating rod, wire clip, short circuit, ground wire, ground terminal, bus clip, grounding clip composition. There is no corresponding alarm device, cannot effectively prompt the operator whether to wear insulated gloves for grounding operation, but cannot help the workers control the habitual violation. 


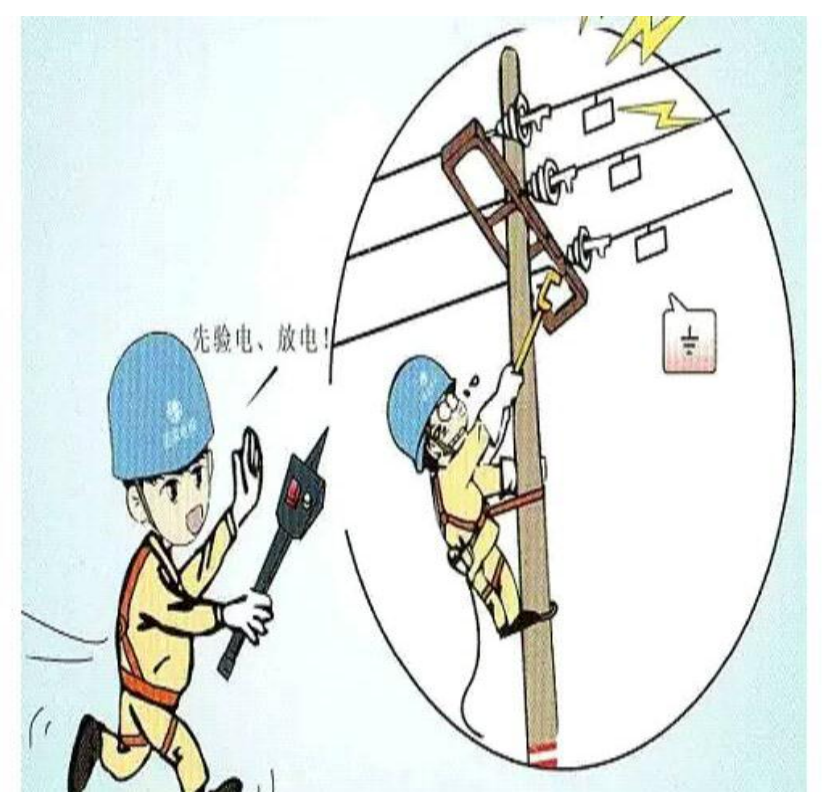

Figure 1

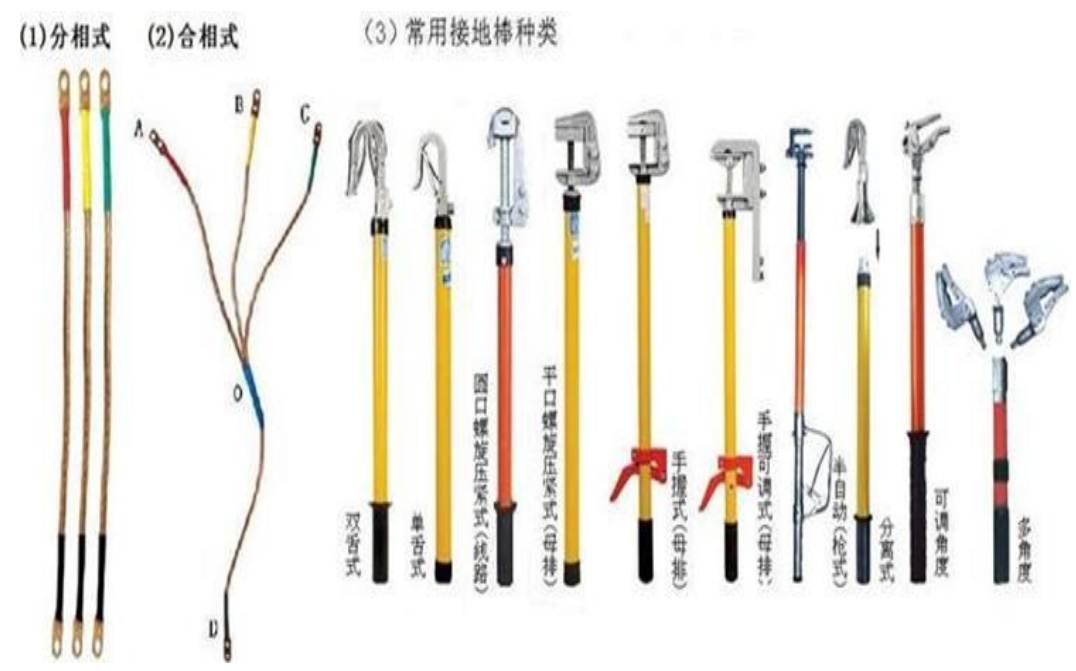

Figure 2

According to the above situation, we designed a hand-held insulation tool detection alarm device, the device has a detachable, can be installed in the grounding rod handle, when the workers wear insulated gloves to operate, the conductivity is 0 , the sensor module does not produce trigger Signal, the gate circuit does not turn on, the light-emitting diode does not shine, the normal ground operation, when the workers empty-handed or not use the specified insulated gloves to operate, because the two electrodes in contact with the hand, the sensor module generates a trigger signal, the gate circuit Through, diode light, prompting the operator to wear insulated gloves. The device has a USB charging function, and can be reminded when the battery is low.

\section{Entire Introduction}

Entire Structure and Insulated Coat Introduction. At present, the use of insulated tools in the substation from the tool room after the use of the state, the location cannot be on-line monitoring, and the market common insulation tools, such as grounding wire insulation rod, the top is the wire clip, The lower part of the handle, cannot effectively prompt the operator whether to wear insulated gloves grounding operation, cannot help the workers control the habitual violation.

The detection alarm device set k60 micro-controller, GPS positioning module, GSM / GPRS communication module, light-emitting diodes. Voice module is equal to one, on the one hand alarm can effectively prevent the occurrence of non-insulated gloves and the emergence of the 
phenomenon of electric shock, on the other hand, and real-time communication with the control center to avoid leakage of grounding, with line closure phenomenon.

This project mainly uses the k60 single chip computer to carry on the overall control, uses the GSM wireless long-distance transmission technology, the GPS localization technology realization localization and the information transmission function, uses the buzzer and the light-emitting diode to play the warning function. When the ground contact is made, the sensor module will generate a trigger when the ground electrode is in contact with the hands. When the ground electrode is in contact with the hand, the sensor module will generate a trigger. Signal, the door circuit, diode light, honey began to ring, prompting the operator to wear insulated gloves.

We use $7.2 \mathrm{v}$ battery to the device power supply, the device using k60 microcontroller, micro-controller with ADC module that can detect the battery voltage, when the battery is no power can be reminded by the voice module to the device charge; another T60 module in the k60 micro-controller, The TSI module also connects a copper foil with a lead wire to distinguish naked contact from contact with insulated gloves. If the operator does not touch the bare hands as specified, the TSI module can detect the signal and send it to the GPS module for the workplace Positioning, the voice module on the device will also issue a warning, and the LED will flash, and through the GPRS module to upload data.

Mechanical Structure and Design. The mechanical part of the alarm device is relatively simple, mainly used in a waterproof case, to place a variety of electronic devices such as SCM, also has a waterproof function. Use the Velcro to install the device to an insulated tool, made removable, easy to use.

The waterproof junction box of the alarm device is $160 * 45 * 55 \mathrm{~mm}$, the protection grade reaches IP66 or above, even if it suffers heavy rain during the power operation, it will not enter the water, and can be used normally to ensure the safety. On the other hand, the Velcro is low cost, good economic applicability, and can be used repeatedly. It is easy to use and can be installed on any insulation tools. Devices such as single-chip, LED and other components need to use $3.3 \mathrm{v}$ voltage, and GSM / GPRS module needs to use 5v voltage, so we use 7.4V, capacity 1500MAH $2 \mathrm{~S}$ battery. The battery can be recharged, the battery is low, the alarm will sound to remind the operator in a timely manner charge.

Control Part of the Design. K60 single-chip microcomputer good volume, excellent performance, cost-effective, and contains TSI touch sensor can be directly connected to aluminum foil

Convenient for Us to Use, So Conducive To K60 for Overall Control. We use WF-NEO-6M GPS positioning module, through the serial port and USB interface to the microcontroller and PC-side output GPS positioning information, easy to use. SIM900A module used to achieve communication functions, remote control center can be realized monitoring. $5 \mathrm{v}$ power supply can be directly connected to the single-chip, support for text messages, data, MMS, Internet and so on. Comfort card really leads, you can achieve on-site unmanned remote reset, with DTMF function to achieve remote control function.

Analysis of the Working Mode. When the operation of wearing gloves, the conductivity is 0 , the gate circuit does not turn on, the light-emitting diode does not shine, the buzzer does not ring, the workers can perform the appropriate operation; when empty hands ground operation, the two electrodes and hands, The sensor module generates the trigger signal, the gate circuit is turned on, the diode light, honey began to ring, prompting the operator to wear insulated gloves.

\section{Works of Innovation Introduced}

Auto-Sensing Alarm. When the ground contact is made, the sensor module will generate a trigger when the ground electrode is in contact with the hands. When the ground electrode is in contact with the hand, the sensor module will generate a trigger. Signal, the door circuit, diode light, honey began to ring, prompting the operator to wear insulated gloves. 
Positioning Communication Function. The GPS module is installed in the device, which can locate the position of the insulation tool in real time. At the same time, the GSM / GPRS module can send information to the control center to facilitate the positioning and remote control of the control center. After the operation, box

Device Removable. The device is removable using a Velcro, which can be mounted on any insulated tool for easy portability and use.

\section{Conclusions}

The use of the device can effectively control the workers habitual violation, improve the safety factor.

Now the rapid development of power industry, power security is more and more important. In the electric work, the worker carries on the line maintenance time, often uses each kind of safe insulation tool, for instance insulates the pole, the earth rod, the electroscope and so on, these tools not only to completes the work certain function, but also to protect the personal safety . According to safety regulations, workers should wear insulated gloves when using insulated tools. Common insulation tools such as grounding wire insulation rod, upper wire clamp, middle insulating rod and lower handle are not effective. Whether personnel wear insulated gloves for grounding operations, cannot help the workers control the habit of illegal. In addition, the current substation hand-held insulation tools from the tool room after the use of the state, the location cannot be on-line monitoring, control center does not facilitate the overall scheduling and management.

The alarm device can achieve positioning, communication, alarm, small size, easy to carry, greatly facilitate the use of the staff. The successful development of the device can greatly improve the safety factor of the power operation, while the cost is relatively low, can be a large number of applications in power construction, with bright prospects.

\section{References}

[1] Zhang Chenbo. On the transmission and distribution lines in operation and management problems and improvements [J].Guangdong Science and Technology.2009. (22):19-20.

[2] Li Baoli. Yu Xi. Safe operation and maintenance of transmission and distribution lines Technical Analysis[J].Super science.2014.(30):71-72.

[3] Zhao Yingfeng. XLPE power cable insulation condition assessment and self-healing remediation technology[D].Southwest Jiaotong University.2013.

[4] $\mathrm{Hu}$ Wei, Zhang hongge. The basic requirement of the electric safety operation [J]. Electric drive automation, 2015, 2015:54-56 + 60 .

[5] Zhang Fuliang. Electric power security tool cabinet research and development of the control system [D]. Hebei university of engineering, 2010.

[6] $\mathrm{Hu}$ Baoyu. Electric power engineering project safety management research [D]. China university of geosciences (Beijing), 2014.

[7] Zhang Xin. Power operation site monitoring technology and implementation [D]. North China electric power university, 2015.

[8] Zheng Yong kun. Monitoring system of power system grounding line in the r\&d and application [D]. Shandong institute of light industry, 2011.

[9] Zheng Yuhui Zhang Naiyun, Li Nana. Intelligent grounding rods alarm technology to explore and design [J]. Journal of information science and technology, 2015, 4:72 + 74 .

[10] Xu Jizhong. Improvement of temporary grounding rods [J]. Rural electrician, 2011, 05:28. 